\title{
FIXED POINT THEOREMS FOR A SUM OF TWO MAPPINGS IN LOCALLY CONVEX SPACES
}

\author{
P. VIJAYARAJU \\ Department of Mathematics \\ Anna University \\ Madras 600 025, India
}

(Received May 2, 1991 and in revised form March 12, 1992)

\begin{abstract}
Cain and Nashed generalized to locally convex spaces a well known fixed point theorem of Krasnoselskii for a sum of contraction and compact mappings in Banach spaces. The class of asymptotically nonexpansive mappings includes properly the class of nonexpansive mappings as well as the class of contraction mappings. In this paper, we prove by using the same method some results concerning the existence of fixed points for a sum of nonexpansive and continuous mappings and also a sum of asymptotically nonexpansive and continuous mappings in locally convex spaces. These results extend a result of Cain and Nashed.
\end{abstract}

KEY WORDS AND PHRASES. Asymptotically nonexpansive and continuous mappings, uniformly asymptotically regular with respect to a map.

1991 AMS SUBJECT CLASSIFICATION CODES. 47H10, 54H25.

\section{INTRODUCTION.}

Let $K$ be a nonempty closed convex bounded subset of a Banach space $X$. In 1955, Krasnoselskii [6] proved first that a sum $T+S$ of two mappings $T$ and $S$ has a fixed point in $K$, when $T: K \rightarrow X$ is a contraction and $S: K \rightarrow X$ is compact (that is, a continuous mapping which maps bounded sets into relatively compact sets) and satisfies the condition that $T x+S y \in K$ for all $x, y \in K$. Nashed and Wong [7] generalized Krasnoselskii's theorem to sum $T+S$ of a nonlinear contraction mapping $T$ of $K$ into $X$ (that is, $\|T x-T y\| \leq \phi(\|x-y\|)$ for all $x, y \in K$, where $\phi$ is a real valued continuous function satisfying certain condition) and a compact mapping $S$ of $K$ into $X$. Subsequently, Edmunds [4], Reinermann [8] extended Krasnoselskii's theorem to a sum $T+S$ of a nonexpansive mapping $T$ and a strongly continuous mapping $S$ (that is, a continuous mapping from the weak topology of $X$ to the strong topology of $X$ ) when the underlying spaces $X$ are Hilbert spaces and uniformly convex Banach spaces respectively.

Krasnoselskii's theorem was further extended by Cain and Nashed [2] to a sum $T+S$ of a contraction mapping $T$ of a nonempty complete convex subset $K$ of a locally convex space $X$ into $X$ and a continuous mappings $S$ of $K$ into $X$. Sehgal and Singh [9] generalized the above result of Cain and Nashed [2] to a sum $T+S$ of a nonlinear contraction mapping $T$ of $K$ into $X$ and a continuous mapping $S$ of $K$ into $X$. This result generalizes the result of Nashed and Wong [7].

The study of asymptotically nonexpansive mappings concerning the existence of fixed points have become attractive to the authors working in nonlinear analysis, since the asymptotically 
nonexpansive mappings include nonexpansive as well as contraction mappings. Goebel and Kirk [5] introduced the concept of asymptotically nonexpansive mappings in Banach spaces and proved a theorem on the existence of fixed points for such mappings in uniformly convex Banach spaces.

The aim of this paper is to prove fixed point theorems for a sum of nonexpansive and continuous mappings in locally convex spaces. Throughout this paper, let $\boldsymbol{X}$ denote a Hausdorff locally convex linear topological space with a family $\left(p_{\alpha}\right)_{\alpha \in J}$ of seminorms which defines the topology on $X$, where $J$ is any index set.

We recall the following definition.

DEFINITION 1.1. Let $K$ be a nonempty subset of $X$. If $T$ maps $K$ into $X$, then

(a) $T$ is called a contraction [2] if for each $\alpha \in J$, there is a real number $k_{\alpha}$ with $0 \leq k_{\alpha}<1$ such that

$$
p_{\alpha}(T x-T y) \leq k_{\alpha} p_{\alpha}(x-y) \quad \text { for all } x, y \text { in } K .
$$

(b) $T$ is called a nonexpansive if $k_{\alpha}=1$ in (a).

(c) $T$ is called an asymptotically nonexpansive [11] if

$$
p_{\alpha}\left(T^{n} x-T^{n} y\right) \leq k_{n} p_{\alpha}(x-y) \quad \text { for all } x, y \text { in } K
$$

for each $\alpha \in J$ and for $n=1,2, \ldots$, where $\left\{k_{n}\right\}$ is a sequence of real numbers such that $\lim _{n \rightarrow \infty} k_{n}=1$.

It is assumed that $k_{n} \geq 1$ and $k_{n} \geq k_{n+1}$ for $n=1,2, \ldots$.

We introduce the following definition.

DEFINITION 1.2. If $T$ and $S$ map $K$ into $X$, then $T$ is called a uniformly asymptotically regular with respect to $S$ if, for each $\alpha$ in $J$ and $\eta>0$, there exists $N=N(\alpha, \eta)$ such that

$$
p_{\alpha}\left(T^{n} x-T^{n-1} x+S x\right)<\eta \quad \text { for all } n \geq N \text { and for all } x \text { in } K .
$$

EXAMPLE 1.3. Let $X=R$ and $K=[0,1]$.

We define a map $T: K \rightarrow X$ by $T x=1+x$ for all $x$ in $K$.

Then $T^{2} x=T(1+x)=2+x$. By induction, we prove that

$$
T^{n} x=n+x .
$$

We define a map $S: K \rightarrow X$ by $S x=-1$ for all $x$ in $K$.

Therefore $\left|T^{n} x-T^{n-1} x+S x\right|=0$. Hence $T$ is uniformly asymptotically regular with respect to $S$.

REMARK 1.4. $T$ is uniformly asymptotically regular with respect to the zero operator means that $T$ is uniformly asymptotically regular [11]. The following example shown in [11] that uniform asymptotic regularity is stronger than asymptotic regularity.

Let $X=\ell^{p}, 1<p<\infty$ and $K$ denote the closed unit ball in $X$. Define a map $T: K \rightarrow$ by

$$
T x=\left(\xi_{2}, \xi_{3}, \ldots\right) \quad \text { for all } x=\left(\xi_{1}, \xi_{2}, \xi_{3}, \ldots\right) \in K .
$$

\section{MAIN RESULTS.}

We state the following Tychonoff's theorem and Banach's contraction principle which will be used to prove our theorems 2.1 and 2.2 .

THEOREM A [10]. Let $K$ be a nonempty compact convex subset of $X$. If $T$ is continuous mapping of $K$ into itself, then $T$ has a fixed point in $K$. 
THEOREM B [2]. Let $K$ be a nonempty sequentially complete subset of $X$. If $T$ is a contraction mapping of $K$ into itself, then $T$ has a unique fixed point $u$ in $K$ and $T^{n} x \rightarrow u$ for all $x$ in $K$.

The following theorem is an extension of Theorem 3.1 of Cain and Nashed [2] for a sum of contraction and continuous mappings to a sum of certain type of asymptotically nonexpansive mapping $T$ and continuous mapping $S$ in locally convex spaces $X$ by assuming the conditions that $T$ is uniformly asymptotically regular with respect to $S$ and $T^{n} x+S y \in K$ for all $x, y$ in $K$ and $n=1,2, \ldots$ This result is new even in the case of normed linear spaces.

THEOREM 2.1 Let $K$ be a nonempty compact convex subset of $X$. Let $T$ be an asymptotically nonexpansive self-mapping of $K$. Let $S$ be a continuous mapping of $K$ into $X$. Suppose that $T$ is uniformly asymptotically regular self-mapping of $K$ with respect to the mapping $S$ and that $T^{n} x+S y \in K$ for all $x, y \in K$ and $n=1,2, \ldots$. Then $T+S$ has a fixed point in $K$.

PROOF. For each fixed $y$ in $K$, we define a map $H_{n}$ from $K$ to $K$ by

$$
H_{n}(x)=a_{n}\left(T^{n} x+S y\right) \text { for all } x \in K .
$$

where $a_{n}=(1-1 / n) / k_{n}$ and $\left\{k_{n}\right\}$ is an in Definition $1.1(c)$. Since $T$ is asymptotically nonexpansive, it follows that

$$
\begin{aligned}
p_{\alpha}\left(H_{n}(a)-H_{n}(b)\right) & =a_{n} p_{\alpha}\left(T^{n} a-T^{n} b\right) \\
& \leq(1-1 / n) p_{\alpha}(a-b) \quad \text { for all } a, b \text { in } K .
\end{aligned}
$$

Hence $H_{n}$ is a contraction on $K$. By Theorem B, $H_{n}$ has a unique fixed point, say, $L_{n} y$ in $K$.

Therefore

$$
L_{n} y=H_{n}\left(L_{n} y\right)=a_{n}\left(T^{n}\left(L_{n} y\right)+S y\right) .
$$

Let $u, v \in K$ be arbitrary. Then we have

$$
\begin{aligned}
p_{\alpha}\left(L_{n} u-L_{n} v\right) & \leq a_{n} p_{\alpha}\left(T^{n}\left(L_{n} u\right)-T^{n}\left(L_{n} v\right)\right)+a_{n} p_{\alpha}(S u-S v) \\
& \leq(1-1 / n) p_{\alpha}\left(L_{n} u-L_{n} v\right)+a_{n} p_{\alpha}(S u-S v)
\end{aligned}
$$

Therefore

$$
p_{\alpha}\left(L_{n} u-L_{n} v\right) \leq n a_{n} p_{\alpha}(S u-S v) .
$$

Since $S$ is continuous, $L_{n}$ is continuous.

Using Tychonoff's Theorem A, we see that $L_{n}$ has a fixed point, say, $x_{n}$ in $K$. Therefore

$$
x_{n}=L_{n} x_{n}=a_{n}\left(T^{n} x_{n}+S x_{n}\right) .
$$

Hence $x_{n}-T^{n} x_{n}-S x_{n}=\left(a_{n}-1\right)\left(T^{n} x_{n}+S x_{n}\right) \rightarrow 0$ as $n \rightarrow \infty$, since $a_{n} \rightarrow 1$ as $n \rightarrow \infty$ and $K$ is bounded and $T^{n} x+S y \in K$ for all $x, y \in K$.

Since $T$ is uniformly asymptotically regular with respect to $S$, it follows that

$$
T^{n} x_{n}-T^{n-1} x_{n}+S x_{n} \rightarrow 0 \text { as } n \rightarrow \infty \text {. }
$$

From (2.4) and (2.5) we obtain

$$
x_{n}-T^{n-1} x_{n} \rightarrow 0 \text { as } n \rightarrow \infty .
$$


Now,

$$
\begin{aligned}
p_{\alpha}\left(x_{n}-(T+S) x_{n}\right) & \leq p_{\alpha}\left(x_{n}-\left(T^{n}+S\right) x_{n}\right)+p_{\alpha}\left(\left(T^{n}+S\right) x_{n}-(T+S) x_{n}\right) \\
& \leq p_{\alpha}\left(x_{n}-\left(T^{n}+S\right) x_{n}\right)+k_{1} p_{\alpha}\left(T^{n-1} x_{n}-x_{n}\right) .
\end{aligned}
$$

Using (2.4) and (2.6) in (2.7) we get

$$
x_{n}-(T+S) x_{n} \rightarrow 0 \text { as } n \rightarrow \infty .
$$

Since $K$ is compact, there exists a subnet $\left(x_{\beta}\right)$ of the sequence $\left\{x_{n}\right\}$ such that

$$
x_{\beta} \stackrel{\beta}{\rightarrow} u \quad \text { for some } u \in K .
$$

Since $T$ and $S$ are continuous, it follows that

$$
\left(I-(T+S)\left(x_{\beta}\right)\right) \stackrel{\beta}{\rightarrow}(I-(T+S))(u)
$$

and by (2.8) we get

$$
x_{\beta}-(T+S)\left(x_{\beta}\right) \stackrel{\beta}{\rightarrow} 0 .
$$

Since $X$ is Hausdorff, it follows that $(I-(T+S))(u)=0$. Hence $T+S$ has a fixed point $u$ in $K$.

For nonexpansive mapping $T$, the condition that $T$ is uniformly asymptotically regular with respect to the map $S$ is not needed in the following theorem. This theorem is an extension of Theorem 3.1 of Cain and Nashed [2] for a sum of contraction and continuous mappings in locally convex spaces.

THEOREM 2.2. Let $K$ be a nonempty compact convex subset of $X$. Let $T$ be a nonexpansive mapping of $K$ into $X$ and $S$ be a continuous mapping of $K$ into $X$ such that $T x+S y \in K$ for all $x, y \in K$. Then $T+S$ has a fixed point in $K$.

PROOF. For each fixed $y$ in $K$, we define a map $H_{n}$ from $K$ to $K$ by

$$
H_{n}(x)=\lambda_{n}(T x+S y) \text { for all } x \in K,
$$

where $\left\{\lambda_{n}\right\}$ is a sequence of real numbers with $0<\lambda_{n}<1$ and $\lambda_{n} \rightarrow 1$ as $n \rightarrow \infty$.

Proceeding as in the above theorem, we obtain a sequence $\left\{x_{n}\right\}$ in $K$ such that $x_{n}=\lambda_{n}\left(T x_{n}+S x_{n}\right)$. Since $K$ is compact and $\left\{x_{n}\right\} \subset K$, there exists a subset $\left(x_{\beta}\right)$ of the sequence $\left\{x_{n}\right\}$ such that

$$
x_{\beta} \stackrel{\beta}{\rightarrow} x \quad \text { for some } x \text { in } K .
$$

Therefore $x_{\beta}=\lambda_{\beta}\left(T x_{\beta}+S x_{\beta}\right)$. Since $T$ and $S$ are continuous, it follows that $x=(T+S) x$. Hence $T+S$ has a fixed point $x$ in $K$.

The following example shows that the above theorem cannot be deduced from Theorem 2.1 .

EXAMPLE 2.3. Let $X=$ space $(s)$, the space of all sequences of complex number whose topology is defined by the family of seminorms $p_{n}$ defined by

$$
p_{n}(x)=\max \left|\xi_{1}\right| \text { for } x=\left(\xi_{1}, \xi_{2}, \ldots\right) \in X \text { and } n=1,2, \ldots
$$

Let $K=\left\{x \in X:\left|\xi_{j}\right| \leq 1\right.$ for $\left.j=1,2, \ldots\right\}$.

Then $K$ is compact [3, Problem 47, p. 346]. Also $K$ is convex.

We define a map $T$ from $K$ to $K$ by $T x=(3 / 4) x$ for all $x \in K$. Then $T$ is nonexpansive.

We define a map $S$ from $K$ to $K$ by $S y=(1 / 4) y$ for all $y \in K$. Then $S$ is continuous.

If $a, b \in K$, then we have

$$
p_{n}(T a+S b) \leq(3 / 4) p_{n}(a)+(1 / 4) p_{n}(b) .
$$


Therefore $T a+S b \in K$ for all $a, b \in K$.

Suppose that $e_{1}=(1,0, \ldots.) \in K$. Then we have

$$
\begin{gathered}
T\left(e_{1}\right)=(3 / 4,0,0, \ldots .), T^{m-1}\left(e_{1}\right)=\left((3 / 4)^{m-1}, 0,0, \ldots\right) \\
T^{m}\left(e_{1}\right)=\left((3 / 4)^{m}, 0,0, \ldots .\right) \text { and } S\left(e_{1}\right)=(1 / 4,0, \ldots .)
\end{gathered}
$$

Therefore

$$
\begin{aligned}
p_{n}\left(T^{m}\left(e_{1}\right)-T^{m-1}\left(e_{1}\right)+S\left(e_{1}\right)\right) & =\left|(3 / 4)^{m}-(3 / 4)^{m-1}+1 / 4\right| \\
& =\left|(1 / 4)\left(1-(3 / 4)^{m-1}\right)\right| \rightarrow 1 / 4 \text { as } m \rightarrow \infty .
\end{aligned}
$$

Hence $T$ is not uniformly asymptotically regular with respect to $S$.

The following example shows that if the condition $T x+S y$ in $K$ for all $x, y \in K$ of Theorem 2.2 is dropped, then the conclusion of theorem fails.

EXAMPLE 2.4. Let $X=R$ and $K=[0,1]$.

We define a map $T$ from $K$ to $K$ by $T x=x / 2$ for all $x \in K$. Then $T$ is a contraction and hence nonexpansive. We define a map $S$ from $K$ to $K$ by $S y=1$ for all $y \in K$. Then $S$ is continuous.

Suppose that $a=3 / 4, b \in K$. Then $|T a+S b|=11 / 8<1 \notin K$. Therefore $T a+S b \notin K$ for some $a, b \in K$.

If $u$ is a fixed point of $T+S$ in $K$, then $u=T u+S u=(u / 2)+1$ and therefore $u=2 \notin K$. Hence $T+S$ has no fixed point in $K$.

To prove of the following Theorems 2.5 and 2.6, we need the following extension of Tychonoff's Theorem A.

THEOREM C [1, p. 169]. Let $K$ be a nonempty closed convex subset of a locally convex space $X$. If $T$ is a continuous mapping of $K$ into itself such that $T(K)$ is contained in a compact subset of $K$, then $T$ has a fixed point in $K$.

In Theorems 2.5 and 2.6, the compactness of the set $K$ of Theorems 2.1 and 2.2 is replaced by a weaker condition that the set $K$ is a complete and bounded set, but the mappings $T$ and $S$ are required to satisfy additional conditions that $S(K)$ is contained in some compact subsets of $K$ and $(I-T-S)(K)$ is closed.

THEOREM 2.5. Let $K$ be a nonempty complete bounded convex subset of $X$. Let $T$ be an asymptotically nonexpansive self-mapping of $K$. Suppose that $S$ is a continuous mapping of $K$ into $X$ such that $S(K)$ is contained in some compact subset $M$ of $K$. Assume further that $T$ is a uniformly asymptotically regular with respect to $S$ and that $T^{n} X+S y$ in $K$ for all $x, y \in K$ and $n=1,2, \ldots$. If $(I-T-S)(K)$ is closed, then $T+S$ has a fixed point in $K$.

PROOF. Define a map $H_{n}$ as in the proof of Theorem 2.1. Proceeding as in Theorem 2.1, $K$ and $L_{n}$ satisfy all hypotheses of Theorem $\mathrm{C}$, where $L_{n}$ is as in the proof of Theorem 2.1. By Theorem C, $L_{n}$ has a fixed point, say, $x_{n}$ in $K$. Since $(I-T-S)(K)$ is closed, it follows from (2.8) that $0 \in(I-T-S)(K)$. Hence the proof is complete.

THEOREM 2.6. Let $K$ be a nonempty complete bounded convex subset of $X$. Let $T$ be a nonexpansive mapping of $K$ into $X$. Suppose that $S$ is a continuous mapping of $K$ into $X$ such that $S(K)$ is contained in a compact subset $M$ of $K$ and $T x+S y \in K$ for all $x, y \in K$. If $(I-T-S)(K)$ is closed, then $T+S$ has a fixed point in $K$.

PROOF. Define a map $H_{n}$ as in the proof of Theorem 2.2. Proceeding as in the proof of Theorem 2.2 and using Theorem $\mathrm{C}$ instead of Tychonoff's Theorem A, we obtain a sequence $\left\{x_{n}\right\}$ in $K$ such that $x_{n}=\lambda_{n}\left(T x_{n}+S x_{n}\right)$.

Since $\lambda_{n} \rightarrow 1$ as $n \rightarrow \infty$ and $K$ is bounded, it follows that $(I-T-S) x_{n} \rightarrow 0$ as $n \rightarrow \infty$.

Since $(I-T-S)(K)$ is closed, it follows that $0 \in(I-T-S)(K)$. 
Hence the proof is complete.

The following example shows that if the condition $T x+S y$ in $K$ for all $x, y \in K$ of Theorem 2.6 is dropped, then the conclusion of theorem fails.

EXAMPLE 2.7. Let $X=\ell_{2}$ and $K=\{x \in X:\|x\| \leq 1\}$. Define a map $T$ from $K$ to $K$ by $T x=\left(0, \xi_{1}, \xi_{2}, \ldots.\right)$ for all $x \in K$. Then $T$ is an isometry and hence nonexpansive.

Define a map $S$ from $K$ to $K$ by $S y=\left(1-\|y\|^{2}, 0, \ldots.\right)$ for all $y \in K$. Then $S$ is compact and hence $S$ is continuous $S(K)$ is contained in a compact subset of $K$.

Suppose that $a=(1 / 2,0, \ldots),. b=(0, \ldots) \in K$. Then we have

$$
\|T a+S b\|^{2}=1+(1 / 4)=5 / 4 .
$$

Therefore $T a+S b \notin K$ for some $a, b \in K$. Suppose that $x$ is a fixed point of $T+S$ in $K$. Then

$$
x=(T+S) x=\left(1-\|x\|^{2}, \xi_{1}, \xi_{2}, \ldots\right) .
$$

Therefore $\xi_{n}=1=\|x\|^{2}$ for $n=1,2, \ldots$ But $\lim _{n \rightarrow \infty} \xi_{n}=0$. Thus $\lim _{n \rightarrow \infty} \xi_{n}=1-\|x\|^{2}$ and hence $\|x\|=1$. Therefore $x=\left(1-\|x\|^{2}, \ldots\right)=(0, \ldots)$ and so $\|x\|=0$ which contradicts $\|x\|=1$. Thus $T+S$ has no fixed point in $K$.

ACKNOWLEDGemENT. I would like to thank Professor T.R. Dhanapalan for his guidance and encouragement in the preparation of this paper.

\section{REFERENCES}

1. BONSALL, F.F., Lectures on some fixed point theorems of functional analysis, Tata Institute, Bombay, India, 1962.

2. CAIN, G.L. (JR.) \& NASHED, M.Z., Fixed points and stability for a sum of two operators in locally convex spaces, Pacific J. Math. 39 (1971), 581-592.

3. DUNFORD, N. \& SCHWARTZ, J.T., Linear Operator, Part I, General Theory Interscience Publishers, INC, New York, 1958.

4. EDMUNDS, D.E., Remarks on nonlinear functional equations, Math. Ann. 174 (1967), 233-239.

5. GOEBEL, K. \& KIRK, W.A., A fixed point theorem for asymptotically nonexpansive mappings, Proc. Amer. Math. Soc. 35 (1972), 171-174.

6. KRASNOSELSKII, M.A., Two remarks on the method of successive approximations, Uspehi Math. Nauk. 10 (1955), 123-127.

7. NASHED, M.A. \& WONG, J.S.W., Some variants of a fixed point theorem Krasnoselskii and applications to nonlinear integral equations, J. Math. Mech. 18 (1969), 767-777.

8. REINERMANN, J., Fixpunktsätze vom Krasnoselskii-Typ., Math. Z. 119 (1971), 339-344.

9. SEHGAL, V.M. \& SINGH, S.P., A fixed point theorem for the sum of two mappings, Math. Japonica 23 (1978), 71-75.

10. TYCHONOFF, A., Ein Fixpunktsätz, Math. Ann. 111 (1935), 767-776.

11. VIJAYARAJU, P., Fixed point theorems for asymptotically nonexpansive mappings, Bull. Calcutta Math. Soc. 80 (1988), 133-136. 


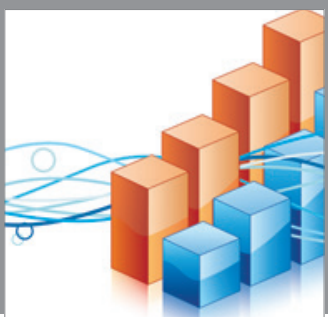

Advances in

Operations Research

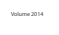

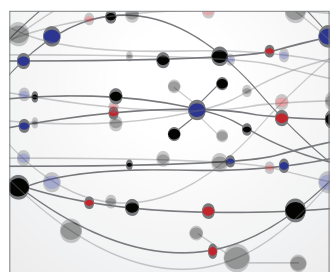

\section{The Scientific} World Journal
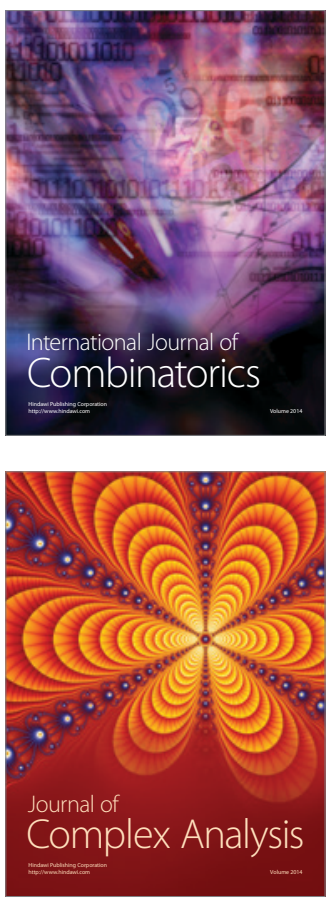

International Journal of

Mathematics and

Mathematical

Sciences
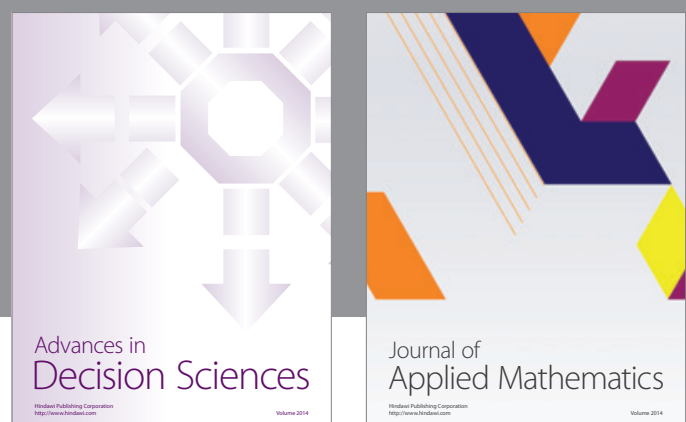

Journal of

Applied Mathematics
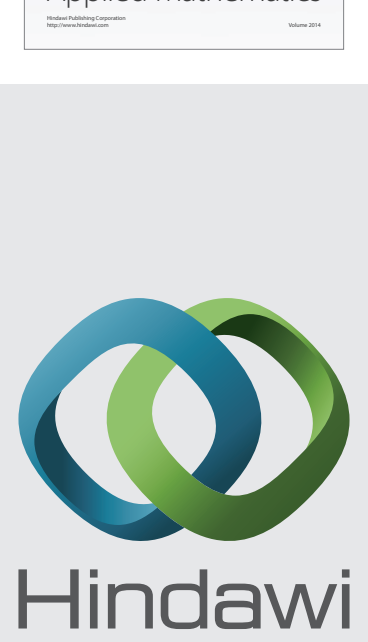

Submit your manuscripts at http://www.hindawi.com
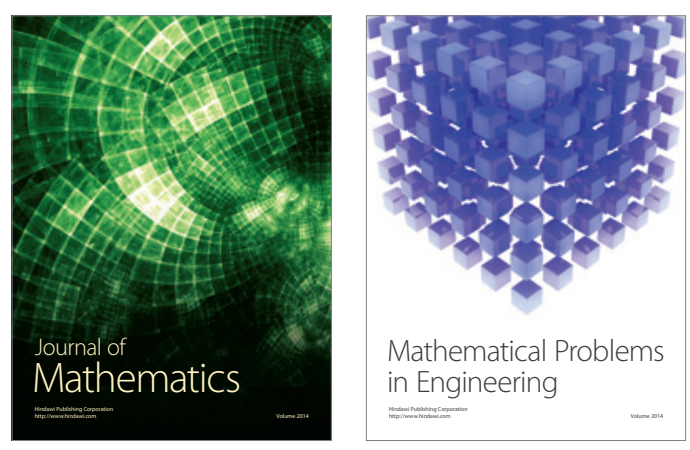

Mathematical Problems in Engineering
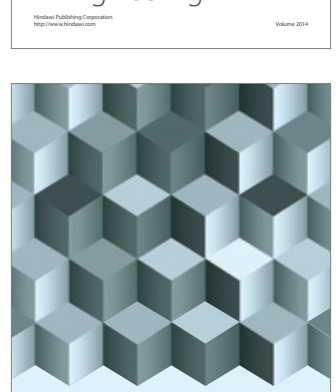

Journal of

Function Spaces
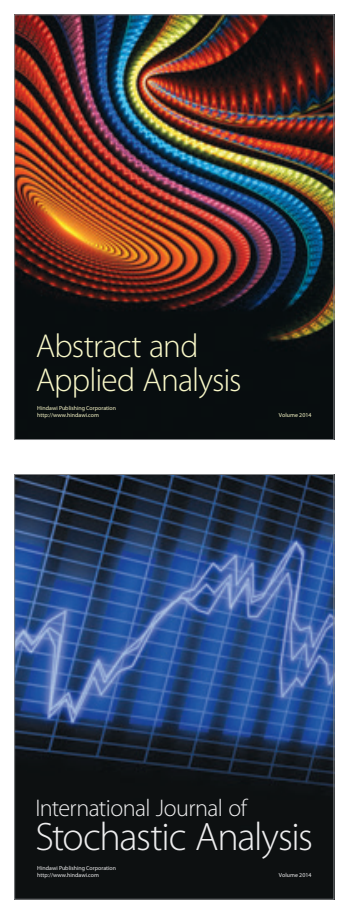

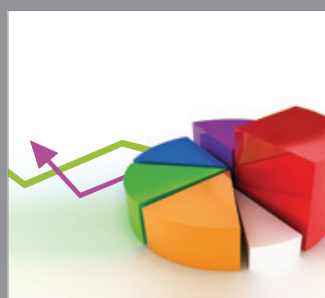

ournal of

Probability and Statistics

Promensencen
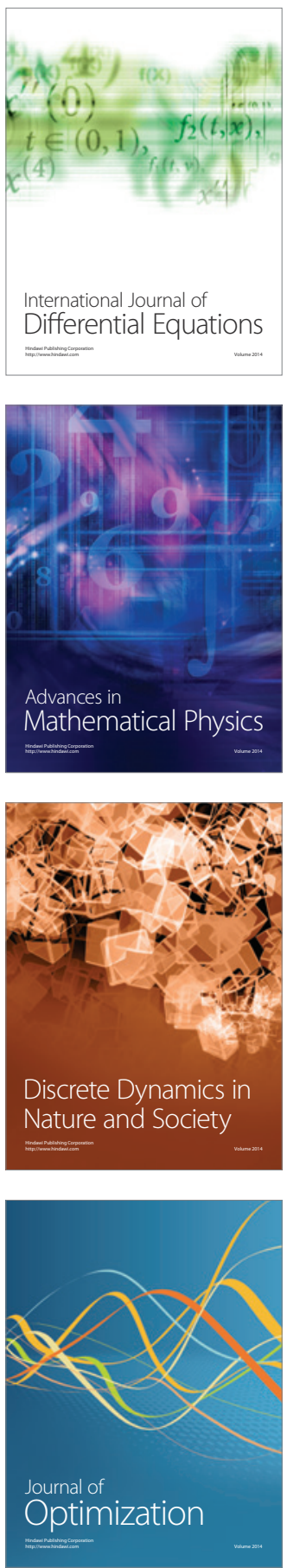